ARTICLE OPEN

\title{
Effect of high-frequency loading and parathyroid hormone administration on peri-implant bone healing and osseointegration
}

\author{
Aya Shibamoto ${ }^{1}$, Toru Ogawa ${ }^{1}$, Joke Duyck ${ }^{2}$, Katleen Vandamme ${ }^{2}$, Ignace Naert ${ }^{2}$ and Keiichi Sasaki ${ }^{1}$
}

The objective of this study is to examine the effect of low-magnitude, high-frequency (LMHF) loading, and anti-osteoporosis medications such as parathyroid hormone (PTH) and bisphosphonates on peri-implant bone healing in an osteoporosis model, and to assess their combined effects on these processes. Thirteen-week-old ovariectomized rats $(n=44)$ were divided into three groups: PTH, alendronate, and saline. After 3 weeks of drug administration, titanium implants were inserted into the tibiae. Each group was subdivided into two groups: with or without LMHF loading via whole-body vibration $(50 \mathrm{~Hz}$ at $0.5 \mathrm{~g}, 15 \mathrm{~min}$ per day, 5 days per week). Rats were killed 4 weeks following implantation. Removal torque test, micro-CT analyses (relative gray (RG) value, water $=0$, and implant $=100$ ), and histomorphometric analyses (bone-to-implant contact $(B I C)$ and peri-implant bone formation (bone volume/tissue volume (BV/TV))) were performed. Removal torque values and BIC were significantly differed by loading and drug administration (ANOVA). Post hoc analysis showed that PTH-treated groups were significantly higher than the other drug-treated groups. BV/TV was significantly enhanced by PTH administration. In cortical bone, RG values were significantly increased by loading. In trabecular bone, however, RG values were significantly increased by PTH administration. These findings suggest that LMHF loading and PTH can act locally and additively on the bone healing process, improving the condition of implant osseointegration.

International Journal of Oral Science (2018)10:6https://doi.org/10.1038/s41368-018-0009-y

\section{INTRODUCTION}

Increasing human life expectancy has meant that greater consideration needs to be given to oral implantation in patients with systemic diseases. Osteoporosis is known as one of the systemic risk factors for implant failure, ${ }^{1,2}$ if without proper management, poor bone quality can result in the lack of primary stability, and impaired bone formation and healing can make achieving osseointegration difficult.

In the field of orthopedics and oral implantology, it is well known that bone adjust its mass and microstructure responding to mechanical loading. Low-magnitude, high-frequency (LMHF) loading, in which a low-magnitude is generally meant as $<1 \mathrm{~g}(1 \mathrm{~g}$ $=9.98 \mathrm{~m} \cdot \mathrm{s}^{-2}$ ) and a high-frequency is generally meant as $20-90 \mathrm{~Hz}$, elicits a positive effect on the skeleton. ${ }^{3,4}$ Numerous studies suggest that LMHF loading, applied by means of whole-body vibration (WBV), stimulates bone formation and fracture healing. ${ }^{5-8}$ WBV loading has been used clinically as a nonpharmacological intervention in the treatment of osteoporosis. ${ }^{9-14}$

With regard to titanium implant osseointegration, which has similarities to bone fracture healing, previous studies have suggested that LMHF loading has an osteogenic effect on periimplant bone. ${ }^{15-17}$ In particular, Ogawa et al. ${ }^{15,18}$ confirmed that the specific parameters of a loading regimen, such as duration, session distribution, frequency, and amplitude of loading, have important roles in the impact of LMHF loading on periimplant bone. In addition, LMHF loading reportedly increased bone-implant osseointegration in osteoporosis models. ${ }^{19-21}$ However, the combined effect of LMHF loading and antiosteoporosis medications, which are the most common treatment of osteoporosis, on peri-implant bone healing and implant osseointegration remains unclear.

Human parathyroid hormone (1-34) [hPTH(1-34)] is a new class of skeletal anabolic agents that stimulates osteoblastic bone formation and has already been approved for the treatment of osteoporotic patients who are at high risk for fractures or who have been intolerant of previous osteoporosis therapy. Intermittent systemic administration of PTH reportedly reduced the risk for fractures by improving bone microarchitecture and enhancing overall bone mass, ${ }^{22-25}$ and exceeded bisphosphonates (BP), which are important antiresorptive agents, in increasing bone mineral density. ${ }^{26}$ Therefore, PTH holds promise as an alternative to existing antiresorptive agents. ${ }^{27}$ There has been growing interest in the use of PTH to accelerate fracture healing and increase bone formation in the early post-operative period after osteosynthesis and following joint replacement with orthopedic implants. ${ }^{28,29}$ The effects of using PTH for maxillofacial bone formation and regeneration have also been investigated in recent years. $^{30}$ Intermittent PTH administration reportedly increased osseointegration of titanium oral implants in animal and clinical models. $^{31-33}$

$\mathrm{BP}$, on the other hand, are antiresorptive agents that reduce osteoclastic bone loss and are widely used for prevention and as

\footnotetext{
${ }^{1}$ Division of Advanced Prosthetic Dentistry, Tohoku University Graduate School of Dentistry, Sendai, Japan and ${ }^{2}$ Department of Oral Health Sciences, Prosthetic Dentistry, BIOMAT -Biomaterials, Katholieke Universiteit Leuven, Leuven, Belgium

Correspondence: Toru Ogawa (ogat-thk@umin.ac.jp)
}

Received: 11 July 2017 Revised: 14 October 2017 Accepted: 16 November 2017

Published online: 13 March 2018 
the first-choice therapy for osteoporosis. BP administration suppress bone resorption and bone remodeling, resulting in a relative increase of bone formation and bone mineral density and subsequently lowing the fracture rate. ${ }^{34}$ The current animal studies have reported its efficacy on implant osseointegration under osteoporotic conditions. ${ }^{35}$

Although both PTH and BP administration are effective for the management of osteoporotic condition, they have some limitations and side effects associated with the prolonged administration, ${ }^{36-38}$ which are possible complications of oral implantation.

Therefore, to treat osteoporotic patients with oral implants safely and successfully, it is necessary to reduce bone healing period and achieve early and strong osseointegration after implant surgery. LMHF loading may be a good consideration for a novel therapeutic option for efficient oral implantation in osteoporotic patients.

This study evaluated the hypothesis that LMHF loading and anti-osteoporosis medications have a beneficial effect on periimplant bone healing and implant osseointegration in osteoporosis model. More specifically, the aim of this study was to investigate the impact of LMHF loading on peri-implant bone

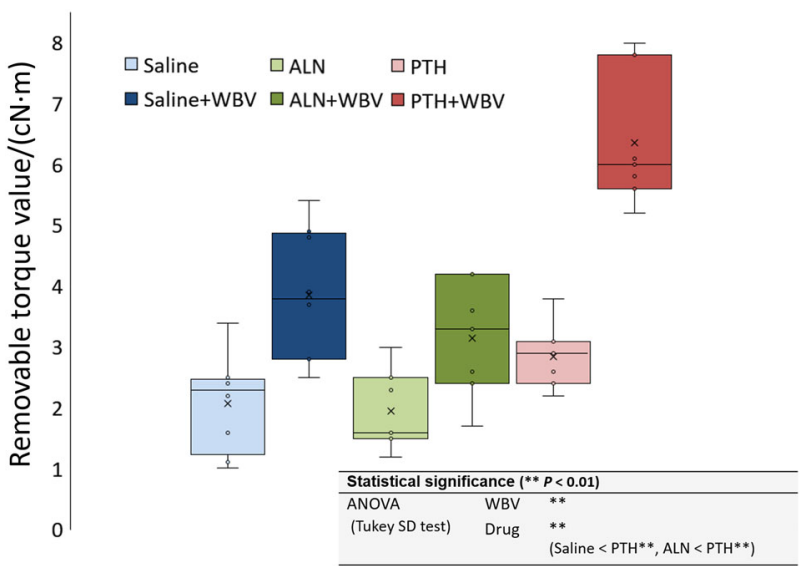

Fig. 1 Results of removal torque (RT) test. The box-plot of the peak loosening torque (RT value), which was measured with a torque gauge. ( ${ }^{* *} P<0.01$; two-way ANOVA followed by Tukey's HSD test). PTH, parathyroid hormone; WBV, whole-body vibration; ALN, alendronate response for ovariectomized (OVX) rats treated with $\mathrm{PTH}$ versus $B P$, to assess which combination is more effective in enhancing implant osseointegration.

\section{RESULTS}

Removal torque test

RT values were significantly differentiated by the loading and the drug administration $(P<0.01)$ (Fig. 1). PTH administration significantly increased the biomechanical strength of the implant-bone interface compared with the saline-treated and alendronate $(A L N)$-treated groups $(P<0.01)$.

\section{Micro-CT analysis}

In the cortical bone surrounding the implant, RG values were significantly affected by the loading $(P<0.01)$ (Fig. 2a). There were no significant differences according to the type of drug. In trabecular bone, RG values of the PTH-treated groups were significantly higher than those of the saline-treated and ALNtreated groups $(P<0.01)$ (Fig. 2b). There were no significant differences according to the loading.

Histologic and histomorphometric analysis

Figure 3 shows representative images of the PTH + WBV and saline groups after 4 weeks of healing. In peri-implant trabecular bone of the drug-treated groups, specifically the PTH-treated groups, bone neoformation was observed. There was little mature bone around the implants in the saline group. In peri-implant cortical bone, the bone width seemed to have thickened by the loading and drug treatments.

In the histomorphometric analysis, BIC was significantly increased by the loading and the PTH administration $(P<0.01)$ (Fig. 4). BV/TV ROI1 and ROI2 were significantly enhanced by the PTH administration $(P<0.01)$ (Fig. 5a, b).

\section{DISCUSSION}

This study investigated the osteogenic impact of LMHF loading and anti-osteoporosis medications on peri-implant bone healing and implant osseointegration in an osteoporosis model, and assessed their combined effects on these processes. The main findings of this study are as follows: (i) LMHF loading and PTH have additive effects on peri-implant bone healing and implant osseointegration in osteoporosis model. (ii) PTH administration is more effective than BP administration on preventing
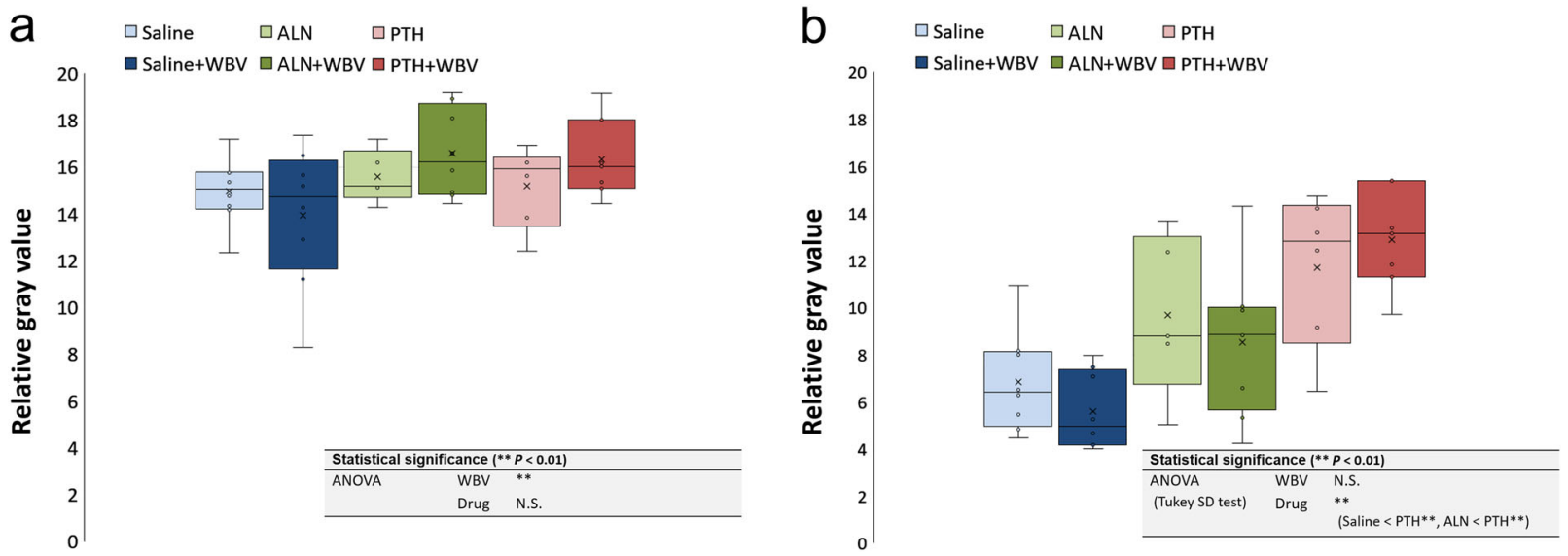

Fig. 2 Results of micro-CT analysis. In a cortical and $\mathbf{b}$ trabecular bone. The box-plot of the relative gray (RG) value (water $=0$ and titanium implant $=100)$ of each region of interest $(\mathrm{ROI})\left({ }^{* *} P<0.01\right.$; two-way ANOVA followed by Tukey's HSD test). PTH, parathyroid hormone; WBV, whole-body vibration; ALN, alendronate 

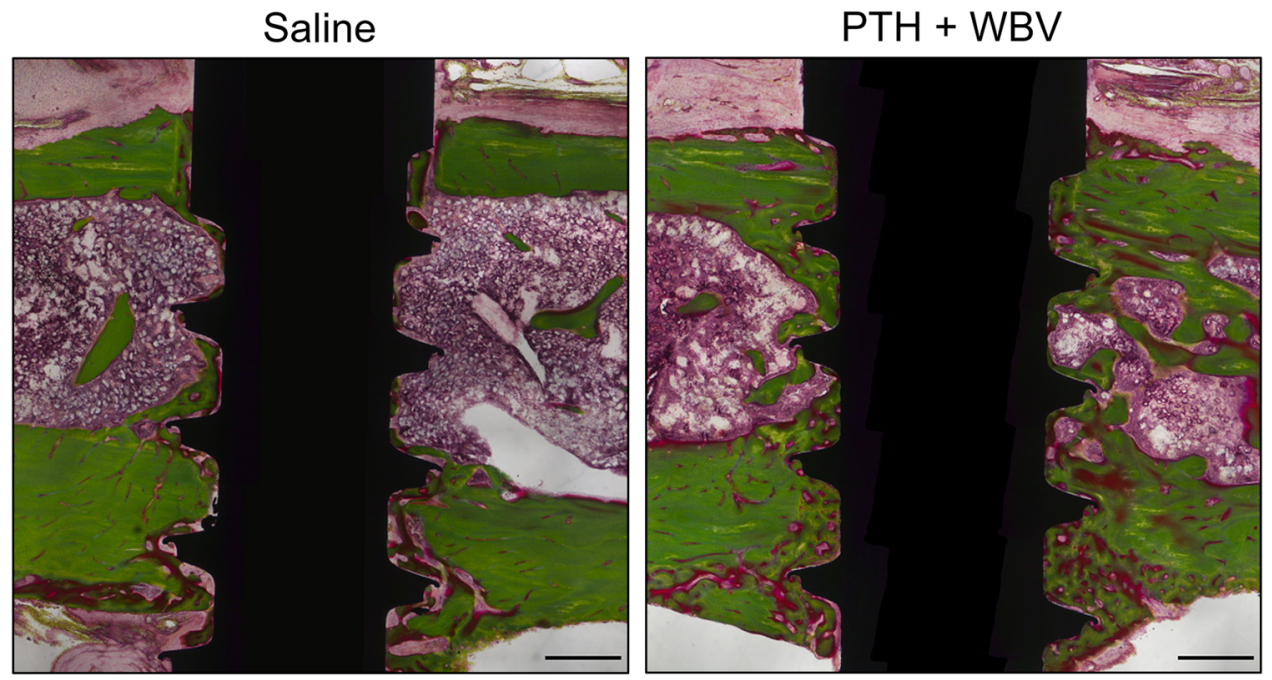

Fig. 3 Representative histological sections stained with Villanueva-Goldner. Histologic sections compared with the test (PTH+WBV) and control (saline) groups after 4 weeks of healing. Scale bar: $500 \mu \mathrm{m}$. PTH, parathyroid hormone; WBV, whole-body vibration



Fig. 4 Histomorphometric results of bone-to-implant contact (BIC). The box-plot of BIC (**P<0.01; two-way ANOVA followed by Tukey's HSD test). PTH, parathyroid hormone; WBV, whole-body vibration; ALN, alendronate

the OVX-induced impaired peri-implant bone response. (iii) LMHF loading and drug administration act locally on the bone healing process.

The assessed bone formation parameters in the PTH + WBV group tended to be highest after 4 weeks of healing, indicating that PTH and WBV additively facilitate implant osseointegration. Interestingly, no obvious positive effect was found in the ALN + WBV groups. Similarly, a comparable study reported that the combination of ALN and LMHF loading did not lead to an additive reaction influencing the bone healing response. ${ }^{21}$ This might be because ALN inhibits osteoclastic bone activity, which is required in the process of bone adaptation and, therefore, implant osseointegration. Collectively, these results indicate that LMHF loading and PTH administration additively increase the osteogenic response in peri-implant bone, achieving early bone healing and strengthening implant osseointegration, and that PTH combined with LMHF loading has a bone-stimulating effect superior to that of ALN and LMHF loading.

Regarding anti-osteoporosis medications, the overall results of the post hoc analysis (Tukey's HSD test) clearly showed that PTH was the medication that exhibited the most pronounced bonestimulating effect compared with ALN and control (saline), based on RT value, BIC, and BV/TV. This affirmed that PTH administration has potent osteogenic capability to stimulate implant osseointegration. Almagro et al. ${ }^{33}$ reported that PTH administration improves implant osseointegration by increasing BIC and peri-implant bone mineral density in a rabbit model of ovariectomy and glucocorticoid-induced osteoporosis, findings that align with the present study. A clinical feasibility study also described the tendency for new bone formation around mini implants obtained from individuals on short-term treatment with teriparatide. ${ }^{32}$ Furthermore, we observed that ALN did not have a positive effect on peri-implant bone formation compared with PTH. These results suggest that such a promotional effect on new bone formation depends on osteoblast activity around implants. ${ }^{39}$ However,

some previous studies reported that BP stimulates implant osseointegration in comparable animal models, ${ }^{35}$ which might be because of using the different osteoporosis animal model or dosing regimen of $\mathrm{BP}$ administration. There are still many controversial aspects regarding this point. Actually, the influence of BP administration on implant success has not been completely determined in clinical practice. ${ }^{40-44}$

Remarkably, micro-CT analysis revealed differences in effect profiles between LMHF loading and PTH. This means that the RG value of cortical bone was increased by the loading. In contrast, the RG value of trabecular bone was increased by the PTH administration. These results suggest that they act locally on the bone healing process. Zhang et al. ${ }^{17}$ reported that only the cortical $\mathrm{BIC}$ significantly increased with high-frequency loading for 4 weeks compared with the unloaded control, suggesting that LMHF loading increased the degree of contact between cortical bone and the implant. Shirota et al. ${ }^{31}$ reported that bone volume density and BIC around implants in the OVX group treated with PTH was almost the same as that of the sham-operated group throughout the observation period. The researchers suggested that intermittent PTH administration not only can prevent resorption of newly generated trabeculae around implants but also can recover bone volume lost because of ovariectomy.

This study has some limitations. The study was planned as a basic study to examine the fundamental phenomenon in osteoporotic models, before progressing to an oral implant model using higher-level experimental animals. As long bones such as the tibia are different from craniofacial bones, further studies using jawbone models are necessary to optimize the performance of vibration devices for local application and explore the optimal timing and duration of PTH administration for peri-implant bone healing and implant osseointegration in osteoporosis. However, the rat tibia, which has been used successfully in previous 

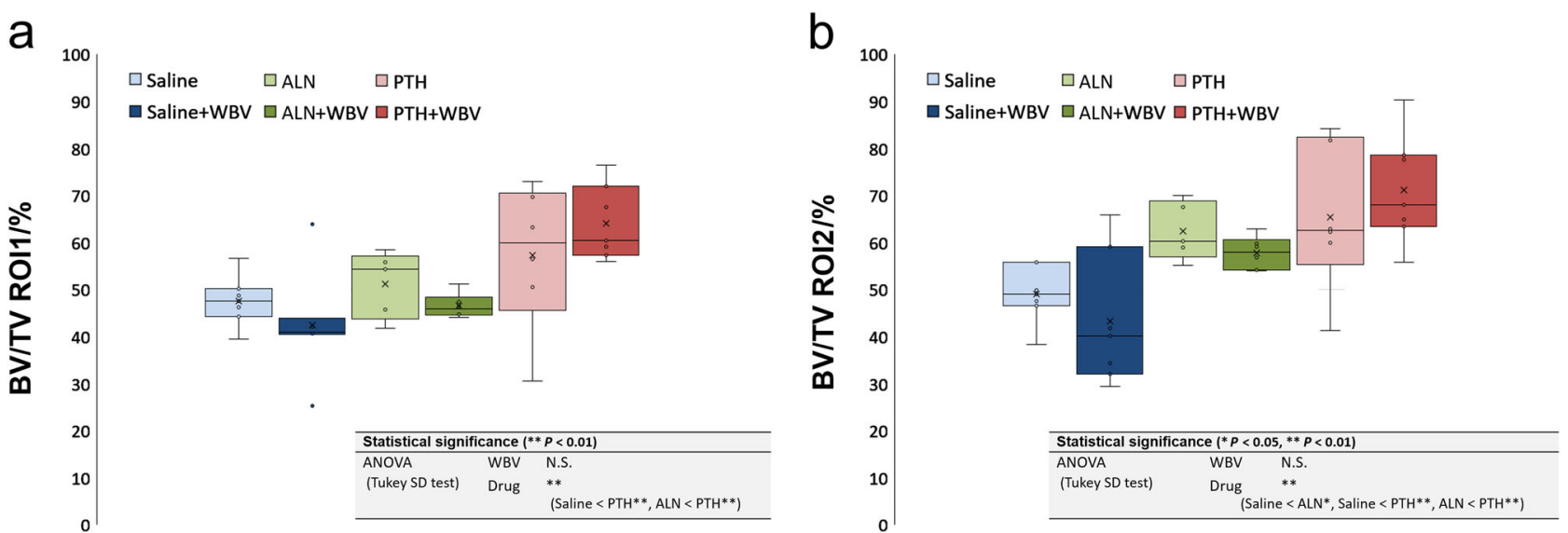

Fig. 5 Histomorphometric results of the bone volume (BV)/tissue volume (TV). The box-plot of the BV/TV of each region of interest (a ROI1 and b ROI2) ( ${ }^{*} P<0.05,{ }^{* *} P<0.01$; two-way ANOVA followed by Tukey's HSD test). PTH, parathyroid hormone; WBV, whole-body vibration; ALN, alendronate
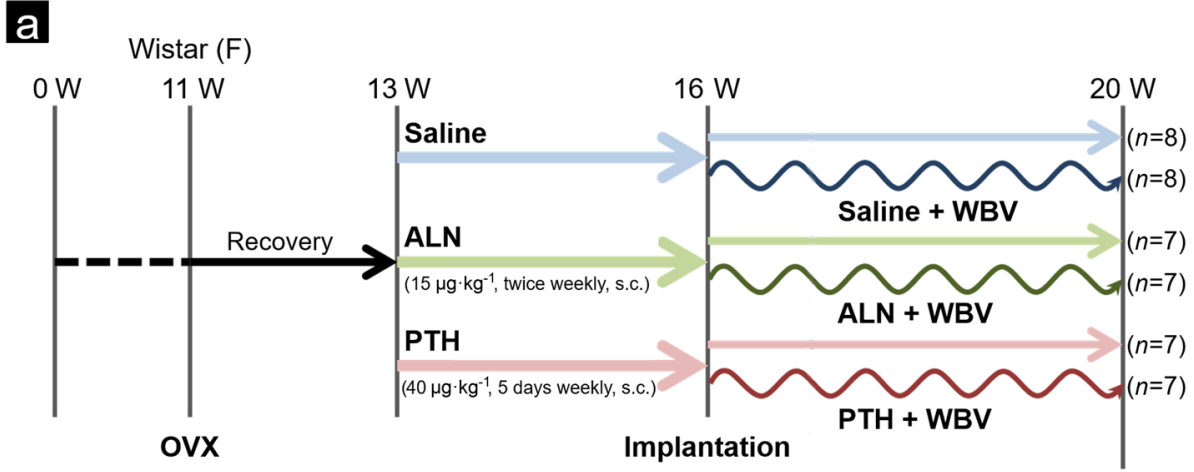

Sacrifice 4 W
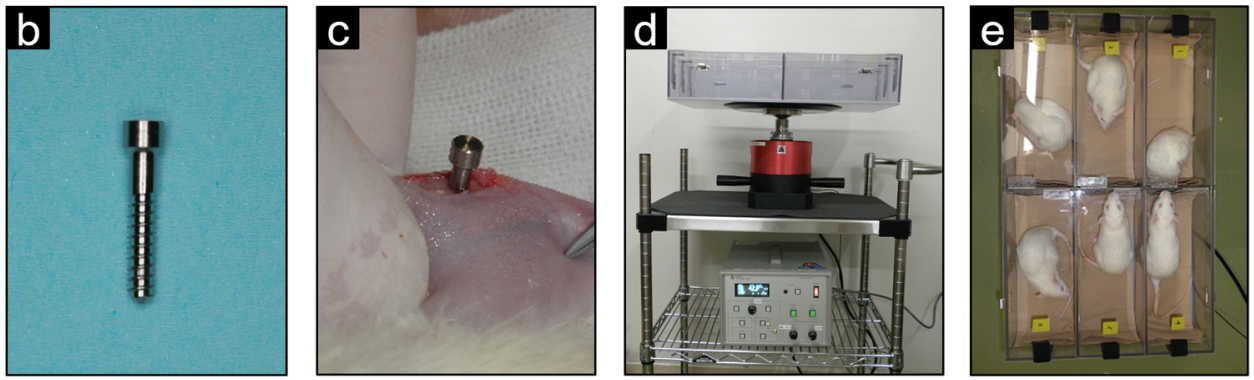

Fig. 6 Experimental protocol and devices. a Illustration of the experimental design and grouping. $\mathbf{b}$ Custom-made titanium implants $(\varnothing: 2 \times$ $13 \mathrm{~mm}^{2}$ ). c The implant was inserted into the proximal metaphysis of the tibiae, perpendicular to the long axis of the tibia. $\mathbf{d}$ Vibration device. e Animal box set onto the device. Six rats could simultaneously undergo vibration loading

experiments, ${ }^{15-18}$ is considered a suitable and reliable location for implant surgery and can be maintained without unpredictable loading. Therefore, the findings of the current study will increase our understanding of the peri-implant bone response to loading and drugs, prior to subsequent studies using an oral implant model.

Osteoporosis could potentially complicate oral implant treatment because of disease-specific characteristics such as abnormal bone conditions and poor bone healing ability. These problems must be resolved by proper management of osteoporotic condition to ensure that oral implant treatment is successful in osteoporotic patients. In summary, the findings of present study suggest that both LMHF loading and intermittent PTH administration have osteogenic potency on peri-implant bone. They also suggest that they act locally and additively on the bone healing process, improving the condition of implant osseointegration. This could be a new therapeutic option for oral implant treatment in osteoporotic patients, avoiding problems such as delayed bone healing and failure of osseointegration.

\section{MATERIALS AND METHODS}

Animals

Forty-four female Wistar rats (age, 11 weeks; average weight, $(171.9 \pm 8.7) \mathrm{g})$ were used in this study. The rats were kept under climate-controlled conditions $\left(23.5^{\circ} \mathrm{C}, 50 \%\right.$ humidity, 12-h light/ dark cycle) and had free access to standard laboratory diet and tap water. 
The study was approved by the Institutional Animal Care and Use Committee of the Tohoku University Environmental \& Safety Committee, and was carried out at the Institute for Animal Experimentation at Tohoku University Graduate School of Medicine.

\section{Experimental design}

Figure $6 a$ illustrates the experimental design. At 11-weeks-old rats were ovariectomized to induce osteoporosis. Two weeks after ovariectomy, rats were divided into six groups: saline $(n=8)$, saline + WBV $(n=8)$, alendronate (ALN) $(n=7), \operatorname{ALN}+$ WBV $(n=$ $7)$, PTH $(n=7)$, and PTH + WBV $(n=7)$. The ALN-treated groups (ALN and ALN + WBV) were injected subcutaneously twice per week with $15 \mu \mathrm{g} \cdot \mathrm{kg}^{-1}$ of ALN (Wako Pure Chemical Industries, Osaka, Japan). The PTH-treated groups (PTH and PTH + WBV) were injected subcutaneously 5 days per week with $40 \mu \mathrm{g} \cdot \mathrm{kg}^{-1}$ of PTH (hPTH(1-34); Peptide Institute, Osaka, Japan). Rats in the saline and saline + WBV groups were administered volume-matched subcutaneous injections of saline. Administration of drugs was continued until the rats were killed. The period from ovariectomy to the initiation of medication was set so that rats had reached the early stages of osteoporosis with the resultant abnormal conditions regulating bone metabolism. ${ }^{45}$ The dosage of ALN was determined by reference to previous studies, ${ }^{46,47}$ and was based on preclinical studies in OVX rats that demonstrated a significant increase in bone mass and strength. ${ }^{48}$ The dosage is comparable with the $20 \mathrm{mg}$ per day dosage prescribed to treat osteoporosis on a $\mathrm{mg}^{-\mathrm{kg}^{-1}}$ basis. The dosage of PTH was determined by reference to a previous study, ${ }^{31}$ and lies within the dose range investigated in a dose-dependency study. ${ }^{49}$ These relatively high dosages were chosen to maximize the possibility of bone-implant osseointegration becoming established.

Three weeks following the onset of drug administration (5 weeks post ovariectomy), a custom-made titanium implant $\left(2 \times 13 \mathrm{~mm}^{2} ;\right.$ cp-Titanium Grade 2, machine surface) was inserted into the proximal metaphysis of both tibiae in rats in all six groups (Fig. 6b, c). Rats were anaesthetized with $2.5 \%$ isoflurane (Escain; Mylan, Pittsburgh, PA, USA). Both cortices of the tibia were perforated at a low rotational speed under constant saline cooling with a surgical drill, which was $0.2 \mathrm{~mm}$ smaller than the implant's diameter to achieve good primary stability. The implants were inserted by means of a custom-fit wrench with manual torque. After implant insertion, wounds were closed using 4-0 polyglycolic acid sutures (Matsuda lka Kogyo, Tokyo, Japan).

In the three groups of rats that underwent WBV (saline + WBV, ALN + WBV, and PTH + WBV), LMHF loading was applied starting the day after implant installation via WBV at a frequency of $50 \mathrm{~Hz}$ and a magnitude of $0.5 \mathrm{~g}$ for $15 \mathrm{~min}$ per day, 5 days per week. Big Wave G-Master (Asahi Seisakusyo, Tokyo, Japan) was used as the vibration device (Fig. 6d, e).

Rats were killed 4 weeks following implantation by cervical displacement under isoflurane-induced anesthesia. The tibiae with the implants were then dissected.

Evaluation of implant osseointegration

Removal torque test: One implant per rat was used to perform the removal torque test. The tibiae were fixated with a jig that was perpendicular to the axis of the implant. A torque gauge (ATG1.5CN/ATG12CN; Tohnichi Mfg, Tokyo, Japan) was attached to the implant head. The peak loosening torque (i.e., the removal torque (RT) value) was recorded.

Micro-CT analysis: Another implant per rat was used for micro-CT analysis. Each sample, positioned in a water-filled plastic cylinder $\left(29 \times 57 \mathrm{~mm}^{2}\right)$, was scanned using a micro-CT system (ScanXmateD225RSS270; Comscantecno, Kanagawa, Japan), which was set to $200 \mathrm{kV}$ and $100 \mu \mathrm{A}$. Three-dimensional sectional images were reconstructed with $928 \times 736$ pixel resolution, in which the pixel
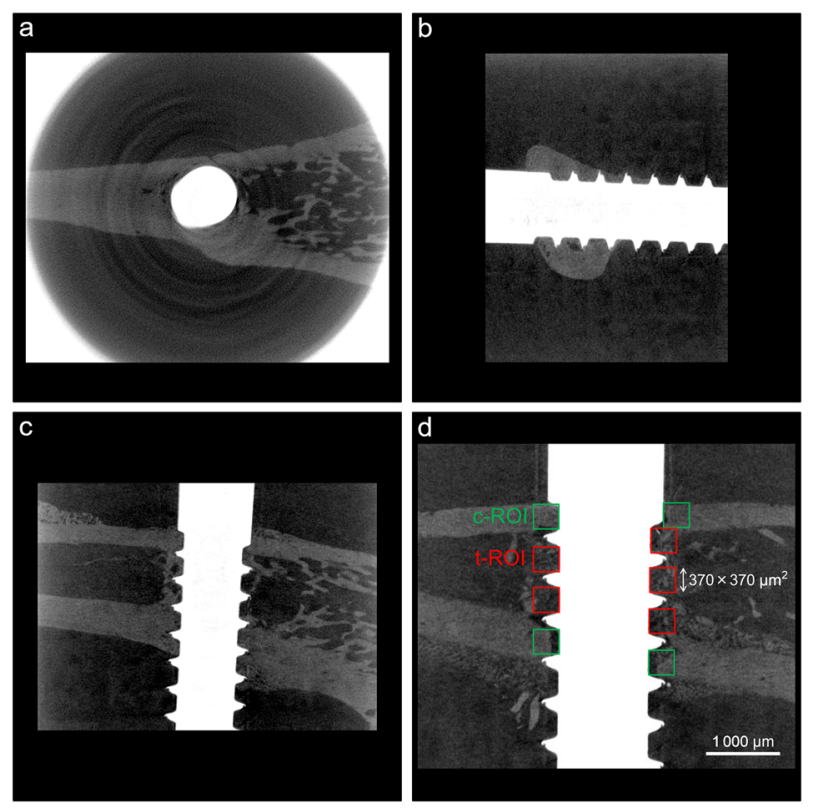

Fig. 7 Evaluation methods of micro-CT analysis. a Axial, b coronal, and $\mathbf{c}$ sagittal images of three-dimensional multiplanar reconstruction (3D MPR). d Illustration of the region of interest (ROI) for microCT analysis. (c-ROI and t-ROI: $0.37 \times 0.37 \mathrm{~mm}^{2}$ (26-pixel) square in the cortical and trabecular bone adjacent to the implant surface)

size was $0.254 \mathrm{~mm}$ and the isotropic voxel size was $10.68 \mu \mathrm{m}$ (Fig. 7a-c). From each 3D multiplanar reconstruction (MPR) images data set, a sagittal slice along the axis of the tibia and implant was selected at the center of the implant as 32-bit grayscale image data. A 32-bit gray value from 0 to 255 was measured using ImageJ software (U.S. National Institutes of Health, Bethesda, MA, USA; http://imagej.nih.gov/ij/, 1997-2014). The region of interest (ROI) was set as a $0.37 \times 0.37 \mathrm{~mm}^{2}$ (26-pixel) square in the cortical and trabecular bone adjacent to the implant surface (c-ROI and t-ROI). A relative gray $(R G)$ value of the cortical and trabecular bone was calculated whereby the gray value of the water and implant was defined as the reference value ( 0 and 100, respectively) (Fig. $7 d$ ).

Histologic and histomorphometric analyses: After micro-CT analysis, the bone-implant blocks were fixed in phosphatebuffered formalin solution and dehydrated in a series of increasing concentrations of alcohol. After dehydration, samples were embedded in poly(methyl methacrylate). Embedded samples were cut using a diamond saw (Exakt BS-300CP; Exakt Technologies, Norderstedt, Germany) along the axis of the tibia and implant. After polishing to a final sample thickness of $40 \mu \mathrm{m}$ (Exakt MG-400CS; Exakt Technologies), sections were stained with Villanueva-Goldner stain.

Histologic and histomorphometric analyses were performed using a light microscope at a magnification of $\times 100$ (Leica DM3000; Leica Laborlux, Wetzlar, Germany). Samples were scanned with a high-sensitivity camera (Leica DFC295; Leica Laborlux) at an image resolution of $1.28 \mu \mathrm{m}$ per pixel. Histomorphometric analysis was performed via digital imaging processing (Adobe Photoshop CS6; Adobe Systems, San Jose, CA, USA and ImageJ, U.S. National Institutes of Health). The following analyses were performed: (i) Bone-to-implant contact (BIC; \%): (summation of the length of contact between the bone and implant $(\mu \mathrm{m}) /$ implant length extending from the most medial to most lateral BIC point $(\mu \mathrm{m})) \times 100$; and (ii) Peri-implant bone volume relative to tissue volume (BV/TV; \%): (area occupied by bone $\left(\mu \mathrm{m}^{2}\right) /$ reference area $\left.\left(\mu \mathrm{m}^{2}\right)\right) \times 100$. Two regions of interest (ROI) were defined: 0-100 $\mu \mathrm{m}$ (BV/TV ROI1) and 100-500 $\mu \mathrm{m}$ (BV/TV ROI2) zones extending from the implant surface. The areas encompassed the 




Fig. 8 Evaluation methods of histomorphometric analysis. Illustration of the reference sites (ROI1: 0 (implant surface)-100 $\mu \mathrm{m}, \mathrm{ROI2:}$ $100-500 \mu \mathrm{m})$ for the bone volume (BV)/tissue volume (TV) evaluation

peri-implant tissues from the medial to lateral cortex (Fig. 8). BIC and BV/TV measurements were performed on both the proximal and distal implant sides.

Statistical analysis

Statistical analyses were conducted using SPSS (ver. 13.0; SPSS Inc., Chicago, IL, USA). Two-way analysis of variance was performed to evaluate differences among the drug administration (ALN, PTH, or saline) and the loading (with or without WBV). The Tukey's honest significant difference (HSD) test was performed to compare differences between groups. A $P$-value $<0.05$ was considered statistically significant.

\section{ACKNOWLEDGEMENTS}

This study was partially supported by a Grant-in-Aid for Scientific Research (C) (15K11147), a Grant-in-Aid for Challenging Exploratory Research (26670830), and a Grant-in-Aid for Young Scientists (B) (26861620) from the Ministry of Education, Culture, Sports, Science and Technology, Japan. We thank Dr. Masahiro likubo, Department of Oral Diagnosis, Tohoku University Graduate School of Dentistry, for providing assistance with the micro-CT analysis.

\section{ADDITIONAL INFORMATION}

Competing interests: The authors declare no competing interests.

\section{REFERENCES}

1. Hwang, D. \& Wang, H. L. Medical contraindications to implant therapy: part I: absolute contraindications. Implant. Dent. 15, 353-360 (2006).

2. Hwang, D. \& Wang, H. L. Medical contraindications to implant therapy: part II: relative contraindications. Implant. Dent. 16, 13-23 (2007).

3. Rubin, C., Turner, A. S., Bain, S., Mallinckrodt, C. \& McLeod, K. Anabolism. Low mechanical signals strengthen long bones. Nature 412, 603-604 (2001).

4. Judex, S., Gupta, S. \& Rubin, C. Regulation of mechanical signals in bone. Orthod. Craniofac. Res. 12, 94-104 (2009).
5. Omar, H. et al. Effect of low magnitude and high frequency mechanical stimuli on defects healing in cranial bones. J. Oral. Maxillofac. Surg. 66, 1104-1111 (2008).

6. Hwang, S. J., Lublinsky, S., Seo, Y. K., Kim, I. S. \& Judex, S. Extremely smallmagnitude accelerations enhance bone regeneration: a preliminary study. Clin. Orthop. Relat. Res. 467, 1083-1091 (2009).

7. Sehmisch, S. et al. Effects of low-magnitude, high-frequency mechanical stimulation in the rat osteopenia model. Osteoporos. Int. 20, 1999-2008 (2009).

8. Shi, H. F., Cheung, W. H., Qin, L., Leung, A. H. \& Leung, K. S. Low-magnitude highfrequency vibration treatment augments fracture healing in ovariectomyinduced osteoporotic bone. Bone 46, 1299-1305 (2010).

9. Russo, C. R. et al. High-frequency vibration training increases muscle power in postmenopausal women. Arch. Phys. Med. Rehabil. 84, 1854-1857 (2003).

10. Rubin, C. et al. Prevention of postmenopausal bone loss by a low-magnitude, high-frequency mechanical stimuli: a clinical trial assessing compliance, efficacy, and safety. J. Bone Mineral. Res. 19, 343-351 (2004).

11. Verschueren, S. M. et al. Effect of 6-month whole body vibration training on hip density, muscle strength, and postural control in postmenopausal women: a randomized controlled pilot study. J. Bone Mineral Res. 19, 352-359 (2004).

12. Iwamoto, J., Takeda, T., Sato, Y. \& Uzawa, M. Effect of whole-body vibration exercise on lumbar bone mineral density, bone turnover, and chronic back pain in post-menopausal osteoporotic women treated with alendronate. Aging Clin. Exp. Res. 17, 157-163 (2005).

13. Gusi, N., Raimundo, A. \& Leal, A. Low-frequency vibratory exercise reduces the risk of bone fracture more than walking: a randomized controlled trial. BMC MusCuloskelet. Disord. 7, 92 (2006).

14. von Stengel, S., Kemmler, W., Engelke, K. \& Kalender, W. A. Effects of whole body vibration on bone mineral density and falls: results of the randomized controlled ELVIS study with postmenopausal women. Osteoporos. Int. 22, 317-325 (2011).

15. Ogawa, T. et al. Influence of whole-body vibration time on peri-implant bone healing: a histomorphometrical animal study. J. Clin. Periodontol. 38, 180-185 (2011).

16. Ogawa, T. et al. The effect of whole-body vibration on peri-implant bone healing in rats. Clin. Oral. Implants Res. 22, 302-307 (2011).

17. Zhang, X. et al. Enhancement of implant osseointegration by high-frequency lowmagnitude loading. PLOS ONE 7, e40488 (2012).

18. Ogawa, T. et al. Stimulation of titanium implant osseointegration through highfrequency vibration loading is enhanced when applied at high acceleration. Calcif. Tissue Int. 95, 467-475 (2014).

19. Akca, K. et al. Micro-morphologic changes around biophysically stimulated titanium implants in ovariectomized rats. Head Face Med. 3, 28 (2007).

20. Chen, B., Li, Y., Xie, D. \& Yang, X. Low-magnitude high-frequency loading via whole body vibration enhances bone-implant osseointegration in ovariectomized rats. J. Orthop. Res. 30, 733-739 (2012).

21. Chatterjee, $M$. et al. High-frequency loading positively impacts titanium implant osseointegration in impaired bone. Osteoporos. Int. 26, 281-290 (2015).

22. Neer, R. M. et al. Effect of parathyroid hormone (1-34) on fractures and bone mineral density in postmenopausal women with osteoporosis. N. Engl. J. Med. 344, 1434-1441 (2001).

23. Aspenberg, P. et al. Teriparatide for acceleration of fracture repair in humans: a prospective, randomized, double-blind study of 102 postmenopausal women with distal radial fractures. J. Bone Mineral. Res. 25, 404-414 (2010).

24. Hodsman, A. B., Kisiel, M., Adachi, J. D., Fraher, L. J. \& Watson, P. H. Histomorphometric evidence for increased bone turnover without change in cortical thickness or porosity after 2 years of cyclical hPTH(1-34) therapy in women with severe osteoporosis. Bone 27, 311-318 (2000).

25. Jiang, Y. et al. Recombinant human parathyroid hormone (1-34) [teriparatide] improves both cortical and cancellous bone structure. J. Bone Mineral. Res. 18, 1932-1941 (2003).

26. Black, D. M. et al. The effects of parathyroid hormone and alendronate alone or in combination in postmenopausal osteoporosis. N. Engl. J. Med. 349, 1207-1215 (2003).

27. Hodsman, A. B. et al. Parathyroid hormone and teriparatide for the treatment of osteoporosis: a review of the evidence and suggested guidelines for its use. Endocr. Rev. 26, 688-703 (2005).

28. Chintamaneni, S., Finzel, K. \& Gruber, B. L. Successful treatment of sternal fracture nonunion with teriparatide. Osteoporos. Int. 21, 1059-1063 (2010).

29. Skripitz, R. \& Aspenberg, P. Parathyroid hormone-a drug for orthopedic surgery? Acta Orthop. Scand. 75, 654-662 (2004).

30. Chan, H. L. \& McCauley, L. K. Parathyroid hormone applications in the craniofacial skeleton. J. Dent. Res. 92, 18-25 (2013).

31. Shirota, T., Tashiro, M., Ohno, K. \& Yamaguchi, A. Effect of intermittent parathyroid hormone (1-34) treatment on the bone response after placement of titanium implants into the tibia of ovariectomized rats. J. Oral. Maxillofac. Surg. 61, 471-480 (2003). 
32. Kuchler, U., Luvizuto, E. R., Tangl, S., Watzek, G. \& Gruber, R. Short-term teriparatide delivery and osseointegration: a clinical feasibility study. J. Dent. Res. 90 1001-1006 (2011).

33. Almagro, M. I. et al. PTH [1-34] enhances bone response around titanium implants in a rabbit model of osteoporosis. Clin. Oral. Implants Res. 24, 1027-1034 (2013).

34. Iwamoto, J., Takeda, T. \& Sato, Y. Efficacy and safety of alendronate and risedronate for postmenopausal osteoporosis. Curr. Med. Res. Opin. 22, 919-928 (2006).

35. Vohra, F., Al-Rifaiy, M. Q., Almas, K. \& Javed, F. Efficacy of systemic bisphosphonate delivery on osseointegration of implants under osteoporotic conditions: lessons from animal studies. Arch. Oral. Biol. 59, 912-920 (2014).

36. Brown, J. P. et al. Bisphosphonates for treatment of osteoporosis: expected benefits, potential harms, and drug holidays. Can. Fam. Physician 60, 324-333 (2014).

37. Tashjian, A. H. Jr. \& Chabner, B. A. Commentary on clinical safety of recombinant human parathyroid hormone 1-34 in the treatment of osteoporosis in men and postmenopausal women. J. Bone Mineral. Res. 17, 1151-1161 (2002).

38. Vahle, J. L. et al. Skeletal changes in rats given daily subcutaneous injections of recombinant human parathyroid hormone (1-34) for 2 years and relevance to human safety. Toxicol. Pathol. 30, 312-321 (2002).

39. Ohtori, S. et al. Teriparatide accelerates lumbar posterolateral fusion in women with postmenopausal osteoporosis: prospective study. Spine 37, E1464-E1468 (2012).

40. Bell, B. M. \& Bell, R. E. Oral bisphosphonates and dental implants: a retrospective study. J. Oral. Maxillofac. Surg. 66, 1022-1024 (2008).

41. Fugazzotto, P. A., Lightfoot, W. S., Jaffin, R. \& Kumar, A. Implant placement with or without simultaneous tooth extraction in patients taking oral bisphosphonates: postoperative healing, early follow-up, and the incidence of complications in two private practices. J. Periodontol. 78, 1664-1669 (2007).

42. Grant, B. T., Amenedo, C., Freeman, K. \& Kraut, R. A. Outcomes of placing dental implants in patients taking oral bisphosphonates: a review of 115 cases. J. Oral. Maxillofac. Surg. 66, 223-230 (2008).

43. Jeffcoat, M. K. Safety of oral bisphosphonates: controlled studies on alveolar bone. Int. J. Oral. Maxillofac. Implants 21, 349-353 (2006).
44. Zahid, T. M., Wang, B. Y. \& Cohen, R. E. Influence of bisphosphonates on alveolar bone loss around osseointegrated implants. J. Oral. Implantol. 37, 335-346 (2011).

45. Kalu, D. N. The ovariectomized rat model of postmenopausal bone loss. Bone Miner. 15, 175-191 (1991).

46. Fuchs, R. K. et al. Individual and combined effects of exercise and alendronate on bone mass and strength in ovariectomized rats. Bone 41, 290-296 (2007).

47. Campbell, G. M., Bernhardt, R., Scharnweber, D. \& Boyd, S. K. The bone architecture is enhanced with combined PTH and alendronate treatment compared to monotherapy while maintaining the state of surface mineralization in the OVX rat. Bone 49, 225-232 (2011).

48. Rodan, G. A., Seedor, J. G. \& Balena, R. Preclinical pharmacology of alendronate. Osteoporos. Int. 3, S7-S12 (1993).

49. Kneissel, M., Boyde, A. \& Gasser, J. A. Bone tissue and its mineralization in aged estrogen-depleted rats after long-term intermittent treatment with parathyroid hormone (PTH) analog SDZ PTS 893 or human PTH(1-34). Bone 28, 237-250 (2001).

(i) Open Access This article is licensed under a Creative Commons Attribution 4.0 International License, which permits use, sharing, adaptation, distribution and reproduction in any medium or format, as long as you give appropriate credit to the original author(s) and the source, provide a link to the Creative Commons license, and indicate if changes were made. The images or other third party material in this article are included in the article's Creative Commons license, unless indicated otherwise in a credit line to the material. If material is not included in the article's Creative Commons license and your intended use is not permitted by statutory regulation or exceeds the permitted use, you will need to obtain permission directly from the copyright holder. To view a copy of this license, visit http://creativecommons. org/licenses/by/4.0/.

(c) The Author(s) 2018 